\title{
L’héritage social des Jeux olympiques
}

La notion d'héritage des Jeux olympiques et paralympiques est relativement récente et connaît un intérêt croissant depuis les années 2000, notamment dans les recherches scientifiques. Pour Sheu et Preuss (2017), la notion de legacy est un concept de référence pour appréhender les conséquences des Jeux olympiques depuis les Jeux de Londres en 2012 même si elle apparaît dans les projets olympiques dès les années 2000 .

La notion d'héritage est bien plus large que celle d'impact qui était jusqu'alors privilégiée, et renvoie à la fois à des dimensions matérielles et immatérielles; il est difficile d'en circonscrire les contours. L'analyse de la littérature académique révèle les sujets récurrents abordés autour de cette notion (Sheu \& Preuss, 2017). Elle englobe particulièrement le développement urbain, les comportements, les croyances, les connaissances et les réseaux, l'environnement, la gouvernance, etc. Il semble qu'elle soit de plus en plus large et englobante.

Certains auteurs apportent d'ailleurs une vision critique de cette notion, notamment depuis que le CIO en fait son faire-valoir des Jeux. MacAloon (2008) explique que la sémantique du discours sur l'héritage de la «famille olympique» génère une perception positive d'un effort louable. En effet, la notion pousse à porter la focale sur ce qui reste de positif à l'issue des Jeux. De cette manière, de nombreux effets négatifs ne sont pas intégrés dans l'évaluation. Pourtant les outils d'évaluation de l'héritage font l'objet d'une abondante littérature. L'objectif est de mesurer l'effet causal des Jeux olympiques sur un grand nombre de variables de résultats qui recouvrent des thématiques variées: activité économique, emploi, bien-être, pratique sportive, flux touristiques, environnement, etc.

La littérature scientifique a été longtemps focalisée sur l'impact économique des Jeux olympiques et sur leurs aspects urbanistiques. L'héritage social a, quant à lui, plus récemment retenu l'attention (Cashman, 2006; Minnaert, 2012) en même temps que le changement social est devenu un des objectifs de l'organisation des Jeux. Il renvoie lui-même à des dimensions plurielles qui touchent par exemple à la transformation de certaines pratiques, notamment l'engagement des populations dans la pratique physique; il concerne aussi d'autres dimensions, plus intangibles, comme l'adhésion des populations à l'événement et le bonheur ressenti, l'image de la ville-hôte, l'inclusion sociale, etc.

Les différentes contributions rassemblées dans ce numéro permettent d'appréhender à la fois les enjeux méthodologiques liés à l'évaluation de l'héritage social, les controverses que suscitent son appréhension et les perspectives pour mieux le construire. Les différents événements qui permettent de concrétiser ces réflexions s'inscrivent dans un contexte français et international, en s'appuyant sur les Jeux olympiques ou coupe d'Europe de football. La majorité des travaux se projettent vers des événements à venir dont l'héritage est encore en construction, ce qui donne au débat un écho particulier sur la scène publique.

Le premier texte, écrit par D. Charrier, J. Jourdan, H. Bourbillières, M. Djaballah et C. Parmantier, se penche sur l'Euro 2016 de football qui s'est tenu en France. Les auteurs reviennent sur la contradiction entre l'importance que prend la construction d'un héritage social d'un événement sportif et les difficultés à appréhender celui-ci. À travers leurs expériences de recherches, ils définissent ce qu'est l'héritage social à travers cette formule: un «potentiel à renforcer, sur le territoire hôte, la cohésion sociale et la citoyenneté, à générer de la capacitation et de l'éducation, à développer l'activité physique pour tous et la culture sportive, à participer à la structuration des organisations, à valoriser le territoire et à créer des passerelles entre le sport et d'autres champs de l'action publique». À partir de cette définition, ils présentent un dispositif méthodologique qui mobilise à la fois des outils quantitatifs et qualitatifs. La mise en œuvre de ces outils se fait toujours dans des lieux ciblés qui permettent également d'appréhender les effets contrastés de l'héritage social. En effet, un événement sportif ne peut pas avoir un effet global significatif sur l'ensemble du pays, les chercheurs ont choisi d'assurer un suivi plus fin et localisé des effets quels qu'ils soient et quelle que soit leur intensité. Les difficultés à rendre compte des bienfaits sociaux des grands événements sont inversement proportionnelles aux facilités à montrer leurs difficultés économiques. En effet, les méga événements impliquent des budgets de plus en plus importants qui laissent des dettes colossales. Outre cet aspect économique, un certain nombre de nuisances sont associées aux dispositifs de sécurité, aux contraintes de mobilité qui entraînent des saturations du trafic, etc. L'opinion publique est aujourd'hui très sensible à ces contraintes.

H. Bourbillères analyse, dans un deuxième texte, les processus de contestation qui se construisent pour lutter contre les candidatures et leurs conséquences sur la population locale. En s'appuyant sur les exemples des villes européennes qui ont annoncé faire acte de candidature pour les Jeux olympiques de 2024 et qui se sont ensuite 
retirées. Cet échantillon de trois villes (Hambourg, Budapest et Rome) illustre des formes différentes de prise en compte des revendications populaires. Certains organisent des référendums pour une consultation directe de la population. D'autres perçoivent l'opposition quand elle s'organise et se manifeste ou à l'occasion d'une élection. L'opinion publique a une place fondamentale dans toutes candidatures olympiques. La voix des urnes a entraîné l'abandon de nombreux projets au cours des dernières années. Sans une forte adhésion, les nuisances des Jeux seront toujours plus relayées que ses bienfaits. Ce qui nous ramène évidemment à la capacité de l'événement à mobiliser positivement le public à grande échelle.

Dans un troisième texte, cette question est abordée par C. Collinet, M.Delalandre et S. Beaudouin qui se sont penchés sur l'opinion des Français vis-à-vis des Jeux olympiques et paralympiques de Paris en 2024. Le Comité d'organisation pouvait se targuer de scores avantageux dans des sondages d'opinion. Les auteurs ont souhaité approfondir les formes d'engagement et différencier les regards portés par la population en fonction des groupes sociaux. Si les résultats ne démentent pas les faveurs des Français à l'égard des Jeux de Paris, ils révèlent dans quelle mesure certains sujets peuvent susciter des incertitudes, voire inquiéter les habitants, y compris les personnes favorables aux Jeux. Les personnes sont interrogées aussi bien sur les conséquences touristiques, financières qu'urbaines. Leur implication dans l'événement est également interrogée notamment à travers leur souhait de prendre part à l'événement à travers un investissement bénévole.

Dans un quatrième texte, l'effet des Jeux olympiques et paralympiques de Paris est également au cœur de la contribution de R. Richard, A. Marcellini, A. Sakis Pappous, H. Joncheray et $\mathrm{S}$. Ferez qui focalisent leur réflexion sur les personnes en situation de handicap. Les auteurs s'attachent à interroger les conditions de construction d'un héritage immatériel pour Paris 2024. Cette réflexion est particulièrement importante à l'heure où les villes hôtes mettent en avant la disponibilité des infrastructures sportives. L'héritage immatériel est au cœur des préoccupations. Mais il est à la fois difficile à concevoir et à évaluer. Développer une action publique efficiente est donc extrêmement problématique. Les auteurs s'attachent à défendre des dispositifs, en s'appuyant notamment sur l'expérience des Jeux olympiques de Londres en 2012, pour envisager des retombées positives des Jeux de 2024 sur l'inclusion des personnes en situation de handicap. Ils défendent notamment l'importance de la médiatisation des athlètes paralympiques comme levier pour développer plus largement la pratique populaire.

Enfin, le dernier texte de ce dossier offre également une vision prospective sur des Jeux à venir. H. Deng, M. Mancini, L. Zhang et M. Bonino étudient le projet de rénovation urbaine du district de Shougang qui doit accueillir des épreuves des Jeux olympiques d'hiver de Pékin en 2022. Articulée autour du concept de territorialisation, la réflexion montre comment cette friche industrielle doit trouver une nouvelle fonction dans la ville. La transformation de cette zone en espace vert permet d'en faire un outil pour le développement des politiques de santé par un mode de vie actif. Ce projet, révélateur de l'accélération des transformations de la capitale chinoise, est conforme aux attentes du mouvement olympique qui souhaite contribuer à des transformations de la ville au profit d'un environnement propice à l'activité physique pour en tirer des profits sanitaires notamment. Ces deux dernières contributions révèlent les bienfaits espérés des Jeux olympiques, mais la réalisation ou l'optimisation de ces transformations se heurtent souvent à de nombreux obstacles dont les conséquences ne peuvent être évaluées qu'à l'issue du projet.

À travers ce dossier, de nombreux enjeux sont posés en termes d'héritage social des Jeux olympiques. La ville de Paris s'est engagée pour accueillir les Jeux olympiques en 2024 et la construction de cet héritage est au cour de l'action publique qu'elle engage actuellement. Les différentes contributions qui suivent nourrissent la réflexion sur ces enjeux sociétaux majeurs.

Cécile COLLINET ${ }^{*}$ Pierre-Olaf SCHUT

* Auteur correspondant: cecile.collinet@u-pem.fr

\section{Références}

Cashman, R. (2006). The bitter-sweet awakening: the legacy of Sydney 2000 Olympic Games. Petersham (NZ): Walla Walla Press.

MacAloon, J.J. (2008). 'Legacy' as managerial/magical discourse in contemporary olympic affairs. The International Journal of the History of Sport, 25(14), 2060-2071. https:// doi.org/10.1080/09523360802439221.

Minnaert, L. (2012). An Olympic legacy for all? The noninfrastructural outcomes of the Olympic Games for socially excluded groups (Atlanta 1996-Beijing 2008). Tourism Management, 33(2), 361-370. https://doi.org/10.1016/j.tour man.2011.04.005.

Sheu, A., \& Preuss, H. (2017). The legacy of the Olympic Games from 1896-2016. A systematic review of academic publications. Working Paper Series Mainzer Papers on Sport Economics \& Management. Johannes Gutenberg-University Mainz, Faculty of Social Science, Media and Sport, Institute of Sport Science, Department of Sporteconomics \& Sportsociology. https://www.sport.uni-mainz.de/Preuss/Download \%20public/Working\%20Paper\%20Series/Working_Paper_ No_14_Legacy\%20Review.pdf.

Citation de l'article : Collinet C \& Schut P-O (2020) L'héritage social des Jeux olympiques. Mov Sport Sci/Sci Mot, 107, 1-2 